\title{
Treatment quality and outcome for multidrug-resistant tuberculosis patients in four regions of China: a cohort study
}

Xu-Bin Zheng', Vinod K. Diwan², Qi Zhao', Yi Hu' ', Judith Bruchfeld ${ }^{3,4}$, Wei-Li Jiang ${ }^{1}$, Sven Hoffner ${ }^{2}$ and Biao $X u^{1,2^{*}}(D)$

\begin{abstract}
Background: China incurs an extremely low treatment coverage of multidrug-resistant tuberculosis (MDR-TB). This study aimed to understand the experience of MDR-TB patients on quality of health care, and the clinical impact through an up to six-year follow-up.

Methods: Cohorts of MDR-TB patients were built in TB/MDR-TB designated hospitals in four regions of China from 2014 to 2015. Patients were followed up during treatment course, and yearly confirmation afterward until 2019. Delay in MDR-TB diagnosis and treatment was calculated upon bacteriological confirmation and treatment initiation. Risk factors for unfavourable outcomes were identified by multivariate logistic regression.

Results: Among 1168 bacteriological-positive TB patients identified from a 12-million population, 58 (5.0\%) MDR-TB cases were detected. The median delay for MDR-TB diagnosis was 90.0 days, with $13.8 \%$ having a delay above 180.0 days. MDR-TB treatment was only recommended to 19 (32.8\%) participants, while the rest continued with regimen for drug-susceptible TB. In MDR-TB treatment group, 36.8\% achieved treatment success, while the others had incomplete treatment (21.1\%), loss to follow-up (36.8\%) and TB relapse (5.3\%). For non-MDR-TB treatment group, 33.3\% succeeded, $25.6 \%$ relapsed, $2.6 \%$ failed, $23.1 \%$ died, and $15.4 \%$ were lost to follow-up. Overall, only 35.7\% (20/56) of detected MDRTB patients had favourable outcomes and higher education level was positively associated with it (adjusted odds ratio [aOR]: 3.60, 95\% confidence interval [CI]: 1.04-12.5).

Conclusions: A large proportion of patients did not receive MDR-TB treatment and had unfavourable outcomes. Delayed MDR-TB diagnosis resulted in poor quality of MDR-TB care. Rapid diagnosis, regulated patient management and high-quality MDR-TB treatment should be enhanced in China.
\end{abstract}

Keywords: Multidrug-resistant, Tuberculosis, Delay, Treatment, Follow-up, China

\footnotetext{
* Correspondence: bxu@shmu.edu.cn

'Department of Epidemiology, School of Public Health and Key Laboratory of

Public Health Safety (Ministry of Education), Fudan University, 130 Dong An

Road, Shanghai 200032, China

${ }^{2}$ Department of Global Public Health, Karolinska Institutet, Stockholm,

Sweden

Full list of author information is available at the end of the article
}

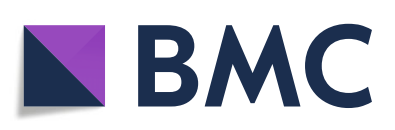

(- The Author(s). 2020 Open Access This article is licensed under a Creative Commons Attribution 4.0 International License, which permits use, sharing, adaptation, distribution and reproduction in any medium or format, as long as you give appropriate credit to the original author(s) and the source, provide a link to the Creative Commons licence, and indicate if changes were made. The images or other third party material in this article are included in the article's Creative Commons licence, unless indicated otherwise in a credit line to the material. If material is not included in the article's Creative Commons licence and your intended use is not permitted by statutory regulation or exceeds the permitted use, you will need to obtain permission directly from the copyright holder. To view a copy of this licence, visit http://creativecommons.org/licenses/by/4.0/. The Creative Commons Public Domain Dedication waiver (http://creativecommons.org/publicdomain/zero/1.0/) applies to the data made available in this article, unless otherwise stated in a credit line to the data. 


\section{Background}

Multidrug-resistant tuberculosis (MDR-TB) has become a global public health crisis, threatening the achievement of "Ending the global TB epidemics" in 2035 [1]. According to the World Health Organization (WHO) Global TB report [2], there were an estimated 500000 new cases having MDR/rifampicin-resistant (RR)-TB in 2018, with an annual increase of over $20 \%$ between 2009 and 2016 [1]. Half of the MDR/RR-TB burden was laid on three countries, i.e., India, China, and the Russian Federation [2]. To fight MDR-TB, globally five priority actions have been proposed, i.e., providing high-quality treatment of drug-susceptible $\mathrm{TB}$, scaling up rapid testing and detection of MDR/RR-TB, ensuring prompt access to effective treatment and proper care, minimizing the risk of disease transmission by quickly enrolling diagnosed patients on effective treatment, and increasing political commitment to ensure necessary financing [3].

China has implemented the modern TB control program since the 1990s, featured by the directly observed treatment, short course strategy (DOTS). With political commitment and pro-poor policy, morbidity and mortality of TB in China have been continuously decreasing at an annual drop of $2.32 \%$ and $7.77 \%$ respectively, from 2000 to 2013 [4]. Although great achievements have been made, China remains the country having the second highest burden of TB and MDR-TB in the world [2]. The challenges to China's MDR-TB control are not only the heavy disease burden but also the poor accessibility and quality of MDR-TB care $[2,5,6]$. As the WHO reported in 2018, the MDR-TB detection rate was only $22.8 \%$ in China coupled with a treatment coverage of $13.6 \%$ [2]. Poor accessibility to drug susceptibility testing (DST) especially in rural areas [7], long waiting time for conventional DST results [8] and incomplete highrisk screening strategy for MDR-TB [9] might explain the low detection rate of MDR-TB in China; while delayed DST, unaffordable treatment cost, drug-induced adverse effects and fragile supply chain of second-line drugs could be the main reasons for the low treatment coverage $[5,10,11]$.

Although several studies reported the difficulties in accessing to MDR-TB diagnosis [7, 12], few have directly illustrated what patients experienced after they were diagnosed as MDR-TB, for instance, what kind of treatment they received and how the quality of care influenced their treatment outcomes. MDR-TB patients should be provided with a recommended regimen containing at least five effective second-line anti-TB drugs in a 6-month intensive phase and at least four drugs in an 18-month consolidation phase according to the national MDR-TB prevention and control program [13], or a standardized shorter MDR-TB regimen as recommended recently by the WHO [14]. Using these guidelines, treatment outcomes can only be assessed in patients who have actually initiated the recommended treatment course. The outcomes of those who did not receive MDR-TB treatment and those treated but lost to follow-up, having incomplete or failed treatment were also critically important from a TB control program perspective. This study was carried out in a cohort of MDR-TB patients in China. The aims of the study were to illustrate the experiences of MDR-TB patients on diagnosis and treatment under China's MDR-TB control program, to identify problems in the provision of high-quality MDR-TB care, and to understand the association between quality of care and treatment outcomes with considerations of demographic and clinical characteristics of patients and molecular features of infected Mycobacteria tuberculosis (M.tb).

\section{Methods \\ Study design}

As a prospective follow-up study, a cohort of MDR-TB patients was conducted in TB/MDR-TB designated hospitals in four geographically varied provinces in China, with two located in east and two in west. The study sites were chosen with considerations of geographic variation, economic level, healthcare infrastructure and TB epidemic status. The population for selected four study sites was 2.0, 3.8, 2.7 and 3.5 million in 2014, respectively. Basic TB care is designated in county or district TB clinics, where sputum smear microscopy is provided for TB diagnosis. Sputum culture was performed for all smear-positive TB patients and for smear-negative TB patients with typical pulmonary lesions on chest X-ray. All positive cultures were submitted to the up-level laboratory in the prefectural Center for Disease Control and Prevention (CDC) or TB hospital for DST, mostly, the conventional DST.

Once the MDR-TB diagnosis was made, patients should be transferred to the designated prefectural TB hospitals for diagnosis confirmation, MDR-TB treatment and patient management. Patients aged > 18 years, confirmed with MDR-TB between January 2014 and December 2015, and providing written inform consent were enrolled in this study. Clinical follow-up was given by clinicians in the designated TB hospitals monthly during the intensive phase, and every other month during the consolidation phase. Additional yearly contact was given from 2014 to 2019 by the healthcare workers in the CDC to follow up patients' TB status and progress.

\section{Microbiological analyses}

Three sputum samples from each patient were collected for direct smear microscopy. Mycobacterial culture was subsequently given to those with positive sputum smear, and those with negative smear but typical pulmonary 
lesions on chest X-ray, using Loewenstein-Jenson (LJ) medium. Species identification and DST were performed for all culture-positive M.tb isolates. Rifampicin, isoniazid, ethambutol, streptomycin, one fluoroquinolone (ofloxacin or levofloxacin) and one second-line injectable drug (kanamycin, capreomycin or amikacin) were included for DST using the proportion method on LJ medium with the following critical concentrations: 40.0, $0.2,2.0,4.0,2.0,2.0,20.0,10.0$, and $4.0 \mathrm{mg} / \mathrm{L}$, respectively [15].

\section{Definitions of treatment regimen and outcomes}

MDR-TB treatment was defined as receiving recommended regimen in the national MDR-TB control program [13]. Non-MDR-TB treatment in this study referred to first-line TB treatment for drug-susceptible $\mathrm{TB}$, which patients had taken before diagnosed as MDR$\mathrm{TB}$, and was kept unchanged after the diagnosis. MDR$\mathrm{TB}$ diagnosis delay was defined as the time period from the diagnosis of smear-positive TB to MDR-TB. TB/ MDR-TB treatment delay was the time period between diagnosis of smear-positive TB/MDR-TB and initiation of TB/MDR-TB treatment. The definition of treatment outcomes depended on the treatment regimen patients received [16]. Cure and treatment completion were defined as favourable outcomes while loss to follow-up, failure, relapse, death and incomplete treatment were uniformly called unfavourable outcomes.

\section{Genotyping and gene sequencing}

Cetyl Trimethyl Ammonium Bromide (CTAB) method was applied to extract DNA from baseline M.tb isolates. Spoligotyping was used to identify the families of the strains and the phylogenetic clades were assigned with reference to SpolDB4 database. Previously reported drug resistance-determining loci [17], including $\operatorname{gyr} A$, $g y r B$, rrs, eis and pncA, were sequenced and mapped to the sequences of published M.tb reference strain $\mathrm{H} 37 \mathrm{Rv}$ to identify the presence or absence of mutations.

\section{Data collection and statistical analysis}

A structured questionnaire was applied to collect sociodemographic and clinical information of participants at the time of TB diagnosis, including age, gender, image of chest $\mathrm{X}$-ray, previous TB treatment history and comorbidities. Details of treatment regimen, sputum culture, DST results, clinical progresses, dates of testing and reporting, and treatment course were extracted from patient's medical chart. After the end of treatment course, yearly follow-up was given to confirm the health condition and TB status. In addition, information in the national TB registration system was exported and matched to the study participants to confirm the TB relapse. Chi-square test and Mann-Whitney U test were applied to identify the differences of socio- demographic and clinical characteristics between patients receiving MDR-TB or non-MDR-TB treatment. Univariate binary logistic regression was used to analyse the factors associated with treatment outcomes, while age, gender and study sites were used for adjustment in multivariate analysis. All statistical analyses were performed with IBM SPSS 22.0 (IBM Corp., Armonk, NY).

\section{Results \\ Study patients}

In total, 1168 bacteriological-positive TB patients, including 1084 smear-positive and 84 smear-negative while culture-positive $\mathrm{TB}$ patients, were diagnosed over the population of 12 million from 2014 to 2015 . Of them, 58 (5.0\%) were later confirmed as MDR-TB. The mean age of MDR-TB patients was 45.4 years and $84.5 \%$ of them were male. There were $19(32.8 \%)$ patients who were transferred to MDR-TB care, while the other 39 (67.2\%) were still under treatment for drug susceptible TB (non-MDRTB treatment group), although 5.1\% (2/39) received an additional fluoroquinolone (FQ). The reasons for not transferring to MDR-TB treatment were: already taking the drug-susceptible TB treatment for a couple of months, effective therapeutic effects judged by clinicians or refusal made by patients. Compared to patients with MDR-TB but not on appropriate treatment, those receiving MDRTB treatment were younger, to a larger proportion of females and from eastern areas, and with a lower coverage of medical insurance $(P<0.05)$, including two patients under a local pro-poor MDR-TB project. (Table 1).

\section{Drug resistance and strain families}

The DST results showed that 31 (53.4\%) M.tb isolates were resistant to isoniazid and rifampicin without additional resistance, 17 (29.4\%) and 5 (8.6\%) isolates had additional resistance to FQs or second-line injectable drugs (SLID), i.e. pre-extensively drug-resistant (pre-XDR), while the remaining five (8.6\%) isolates were resistant to both FQs and SLID and thus defined as XDR. The consistencies between phenotypic and genotypic drug resistance were $73.1 \%$ on isoniazid and $94.2 \%$ on rifampicin. Of the 22 FQs-resistant strains, $16(72.7 \%)$ were detected with mutations in gyrA/gyrB loci, while mutations in rrs/eis loci were observed in $75.0 \%(6 / 8)$ of SLID-resistant strains. Results of spoligotyping showed that 46 (88.5\%) M.tb strains belonged to Beijing family. It was found that non-MDR-TB treatment group had higher proportion of pre-XDR and XDR, and lower proportion of mutations in katG/inhA loci, compared to the group transferred to MDR-TB treatment $(P<0.05)$ (Table 1$)$. 
Table 1 Demographic, clinical and molecular characteristics of MDR-TB patients and isolated Mycobacterium tuberculosis strains ${ }^{\text {a }}$

\begin{tabular}{|c|c|c|c|c|}
\hline \multirow[t]{2}{*}{ Factors } & \multirow[t]{2}{*}{ All patients $(n=58)$} & \multicolumn{2}{|l|}{ Treatment regimen } & \multirow[t]{2}{*}{$P$ value } \\
\hline & & Non-MDR-TB $(n=39)$ & $\operatorname{MDR}-\mathrm{TB}(n=19)$ & \\
\hline Age $^{b}$ & $45.4 \pm 17.3$ & $49.6 \pm 17.6$ & $36.8 \pm 13.4$ & 0.009 \\
\hline Male & $49(84.5)$ & $36(92.3)$ & $13(68.4)$ & 0.047 \\
\hline \multicolumn{5}{|l|}{ Study sites in } \\
\hline Western & $41(70.7)$ & $31(79.5)$ & $10(52.6)$ & \multirow[t]{2}{*}{0.035} \\
\hline Eastern & $17(29.3)$ & $8(20.5)$ & $9(47.4)$ & \\
\hline Body mass index ${ }^{b}$ & $20.4 \pm 2.3$ & $20.7 \pm 2.3$ & $19.8 \pm 2.2$ & 0.191 \\
\hline \multicolumn{5}{|l|}{ Education } \\
\hline Primary school or below & $38(65.5)$ & $25(64.1)$ & $13(68.4)$ & \multirow[t]{2}{*}{0.745} \\
\hline Junior high school or above & $20(34.5)$ & $14(35.9)$ & $6(31.6)$ & \\
\hline Married & $31(53.4)$ & $20(51.3)$ & $11(57.9)$ & 0.636 \\
\hline Health insurance & $51(87.9)$ & $38(97.4)$ & $13(68.4)$ & 0.004 \\
\hline Haemoptysis & $15(25.9)$ & $11(28.2)$ & $4(21.1)$ & 0.752 \\
\hline Tuberculosis treatment history & $25(43.1)$ & $19(48.7)$ & $6(31.6)$ & 0.216 \\
\hline Pulmonary cavities & $28(48.3)$ & 17 (43.6) & $11(57.9)$ & 0.306 \\
\hline \multicolumn{5}{|l|}{ DST pattern } \\
\hline MDR & $31(53.4)$ & 17 (43.6) & $14(73.7)$ & \multirow[t]{2}{*}{0.031} \\
\hline Pre-XDR and XDR & $27(46.6)$ & $22(56.4)$ & $5(26.3)$ & \\
\hline \multicolumn{5}{|l|}{ Genotypic drug resistance $(n=52)$} \\
\hline katG/inhA mutation & $38(73 \cdot 1)$ & $22(62.9)$ & $16(94 \cdot 1)$ & 0.021 \\
\hline rpoB mutation & $49(94 \cdot 2)$ & $32(91.4)$ & $17(100 \cdot 0)$ & 0.542 \\
\hline gyrA gyrB mutation & $20(38.5)$ & $14(40 \cdot 0)$ & $6(35 \cdot 3)$ & 0.744 \\
\hline rrs/eis mutation & $8(15.4)$ & $7(20 \cdot 0)$ & $1(5 \cdot 9)$ & 0.248 \\
\hline pncA mutation & $14(26.9)$ & $11(31.4)$ & $3(17 \cdot 6)$ & 0.341 \\
\hline \multicolumn{5}{|l|}{ Strain family $(n=52)$} \\
\hline Non-Beijing family & $6(11.5)$ & $4(11.4)$ & $2(11.8)$ & \multirow[t]{2}{*}{1.000} \\
\hline Beijing family & $46(88.5)$ & $31(88.6)$ & $15(88.2)$ & \\
\hline
\end{tabular}

DNA was failure to extract from six Mycobacterium tuberculosis isolates. MDR Multidrug-resistant, DST Drug susceptibility testing, XDR Extensively drug-resistant ${ }^{a}$ Data are presented as number (percent) unless other specifies

${ }^{\mathrm{b}}$ Continuous variable; means \pm standard deviations are presented

\section{Diagnosis and treatment delay}

The median delay for MDR-TB diagnosis was 90.0 (interquartile range [IQR]: 64.0-127.5) days with no significant differences between MDR-TB and non-MDRTB treatment groups $(P>0.05)$. Of all participants, $51.7 \%(30 / 58)$ had a diagnosis delay over 90.0 days and $13.8 \%(8 / 58)$ had a delay even over 180.0 days. In terms of treatment delay, the non-MDR-TB treatment group kept their regimen for drug-susceptible TB while the MDR-TB treatment group had a median delay for 7.0 (IQR: 0.0-14.5) days before initiating the recommended treatment. The total delay, summed by diagnosis and treatment delay, for the MDR-TB treatment group was 91.5 (IQR: 83.8-134.0) days (Fig. 1).

\section{Treatment outcomes}

In the non-MDR-TB treatment group, 28 (71.8\%) patients met the requirements for "treatment success" while the rest had treatment failure, death or loss to follow-up at the end of treatment course. In the group transferred to MDR-TB treatment, only 8 (42.1\%) patients had completed their MDR-TB treatment and 7 succeeded. Overall, $71.8 \%$ (28/39) of patients in nonMDR-TB treatment group and 36.8\% (7/19) of patients in MDR-TB treatment group were reported as having favourable outcomes $(P<0.05)$ at the end of their administrated treatment course.

Further yearly follow-up until the end of 2019 disclosed that in the group kept drug-susceptible TB treatment $(n=39)$, only $13(33.3 \%)$ patients remained "treatment success"; 10 (25.6\%) had TB relapse, including 9 previous successfully treated patients; another 4 (10.3\%) died after 


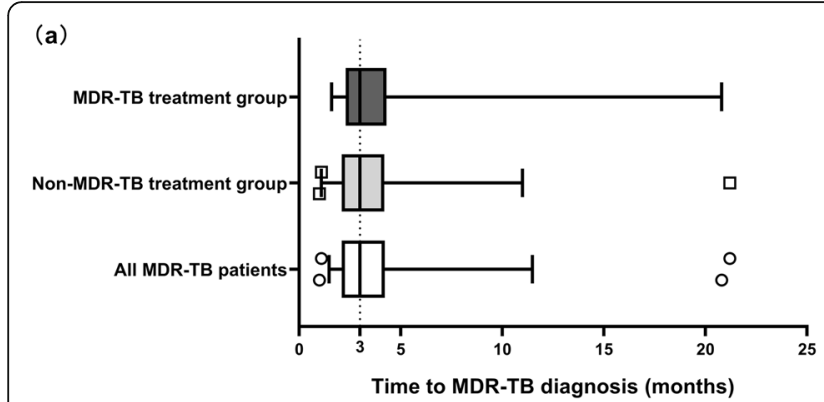

(b)

Fig. 1 Time to multidrug-resistant tuberculosis (MDR-TB) diagnosis and treatment initiation. a Time period from diagnosis of a smear-positive TB to MDR-TB in all MDR-TB patients, patients receiving recommended MDR-TB treatment, and patients continued with the treatment for drugsusceptible TB, namely non-MDR-TB treatment. $\mathbf{b}$ Time period from the diagnosis of smear-positive TB to the initiation of TB treatment in all patients, and time period from the diagnosis of MDR-TB to the initiation of the recommended MDR-TB treatment in those transferred to MDR-TB treatment

reported as "treatment success", of which $50 \%$ had a main cause of death as TB; 1 (2.6\%) did not receive further treatment after failing the previous treatment; and 6 (15.4\%) were lost to follow-up, of which 4 did not complete their treatment and 2 were reported as "treatment success". In the group transferred to MDR-TB treatment, 7 (36.8\%) had treatment success upon follow-up; 1 (5.3\%) had a TB relapse after being successfully treated; 4 (21.1\%) remained untreated, including 2 identified from the loss to follow-up group; and 7 (36.8\%) were lost to follow-up. The treatment success rate among patients who actually completed their treatment was $46.4 \%(13 / 28)$ in non-MDR-TB treatment group, while it was $77.8 \%$ (7/
9) in MDR-TB treatment group. Overall, favourable outcomes were decreased from the previous $71.8 \%(28 / 39)$ to $35.1 \%(13 / 37)$ in patients with non-MDR-TB treatment, while remained to be $36.8 \%$ (7/19) in those transferred to MDR-TB treatment at the last follow-up. The median time for TB relapse after treatment completion was 24.0 (IQR: 12.8-32.5) months and over 90\% occurred within three years (Fig. 2).

\section{Factors associated with treatment outcomes}

Risk factors for unfavourable treatment outcomes at final follow-up were analysed by univariate logistic analysis and followed by multivariate analysis with

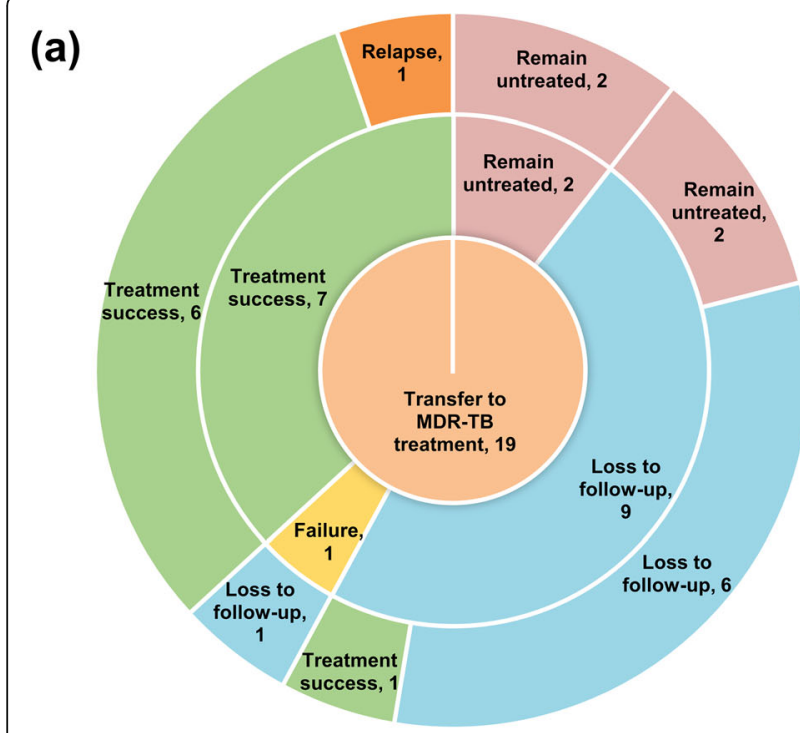

Treatment success Death (b)

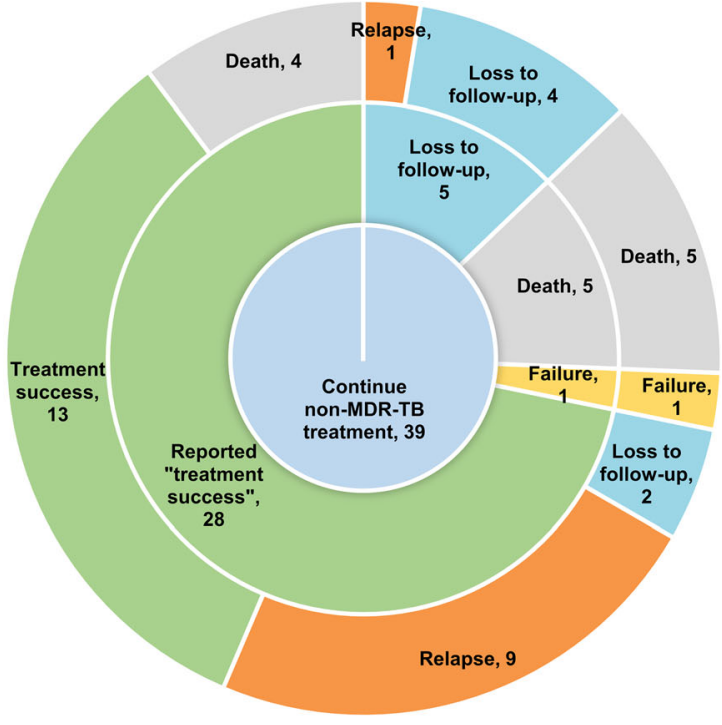

Remain untreated

Loss to follow-up

\section{Treatment failure}

Relapse

Fig. 2 Treatment outcomes for participating multidrug-resistant tuberculosis (MDR-TB) patients receiving MDR-TB treatment (a) and non-MDR-TB treatment $(\mathbf{b})$. The circles from inside to outside represented treatment regimen, treatment outcomes at the end of treatment course, and treatment outcomes at the last follow-up in 2019 
Table 2 Univariate and multivariate analysis of risk factors for unfavourable treatment outcomes among MDR-TB patients

\begin{tabular}{|c|c|c|c|c|}
\hline \multirow[t]{2}{*}{ Factors } & \multicolumn{2}{|c|}{ Treatment outcomes at the last follow-up } & \multirow{2}{*}{$\begin{array}{l}\text { Odds ratio } \\
(95 \% \text { Cl) }\end{array}$} & \multirow{2}{*}{$\begin{array}{l}\text { Adjusted odds } \\
\text { ratio }(95 \% \text { Cl) }\end{array}$} \\
\hline & Favourable outcomes $(n=20)$ & Unfavourable outcomes $(n=36)$ & & \\
\hline Age (mean \pm SD) & $40.8 \pm 16.8$ & $49.1 \pm 16.9$ & $0.97(0.94-1.00)$ & \\
\hline Male & $16(80.0)$ & $31(86.1)$ & $0.65(0.15-2.74)$ & \\
\hline \multicolumn{5}{|l|}{ Study sites in } \\
\hline Western & $13(65.0)$ & $27(75.0)$ & 1 & \\
\hline Eastern & $7(35.0)$ & $9(25.0)$ & $1.62(0.49-5.30)$ & \\
\hline Body mass index (mean \pm SD) & $20.9 \pm 2.0$ & $20.0 \pm 2.4$ & $1.22(0.94-1.58)$ & $1.31(0.99-1.75)$ \\
\hline \multicolumn{5}{|l|}{ Education } \\
\hline Primary school or below & $9(45.0)$ & $28(77.8)$ & 1 & 1 \\
\hline Junior high school or above & $11(55.0)$ & $8(22.2)$ & $4.28(1.31-13.9)$ & $3.60(1.04-12.5)$ \\
\hline Married & $13(65.0)$ & $17(47.2)$ & $2.08(0.67-6.41)$ & $1.66(0.50-5.55)$ \\
\hline Health insurance & $19(95.0)$ & $30(83.3)$ & $3.80(0.42-34.1)$ & $4.54(0.49-42.0)$ \\
\hline Haemoptysis & $4(20.0)$ & $11(30.6)$ & $0.57(0.15-2.10)$ & $0.59(0.15-2.29)$ \\
\hline Tuberculosis treatment history & $6(30.0)$ & $18(50.0)$ & $0.43(0.13-1.36)$ & $0.43(0.13-1.49)$ \\
\hline Pulmonary cavities & $12(60.0)$ & $15(41.7)$ & $2.10(0.69-6.39)$ & $1.92(0.52-7.12)$ \\
\hline \multicolumn{5}{|l|}{ DST pattern } \\
\hline MDR & $12(60.0)$ & $18(50.0)$ & 1 & 1 \\
\hline Pre-XDR and XDR & $8(40.0)$ & $18(50.0)$ & $0.67(0.22-2.02)$ & $0.87(0.27-2.84)$ \\
\hline \multicolumn{5}{|l|}{ Strain family $(n=50)$} \\
\hline Non-Beijing family & $2(10.5)$ & $4(12.9)$ & 1 & 1 \\
\hline Beijing family & $17(89.5)$ & $27(87.1)$ & $1.26(0.21-7.64)$ & $1.58(0.24-10.4)$ \\
\hline \multicolumn{5}{|l|}{ Treatment regimen } \\
\hline Non-MDR-TB treatment & $13(65.0)$ & $24(66.7)$ & 1 & 1 \\
\hline MDR-TB treatment & $7(35.0)$ & $12(33.3)$ & $1.08(0.34-3.40)$ & $0.52(0.13-2.10)$ \\
\hline \multicolumn{5}{|l|}{ MDR-TB diagnosis delay (days) } \\
\hline$\leq 90.0$ & $13(65.0)$ & $18(50.0)$ & 1 & 1 \\
\hline$>90.0$ & $7(35.0)$ & $18(50.0)$ & $0.54(0.17-1.66)$ & $0.44(0.12-1.55)$ \\
\hline
\end{tabular}

DNA was failure to extract from six Mycobacterium tuberculosis isolates. Two patients were once reported as treatment success but were lost at the last yearly follow-up, thus excluded from the analysis. MDR Multidrug-resistant, SD Standard deviation, DST Drug susceptibility testing, XDR Extensively drug-resistant ${ }^{\text {a }}$ Adjusted by age, gender and study sites

adjustment of age, gender and study sites. Two patients from non-MDR-TB treatment group were excluded from analysis due to loss to follow-up after being reported as "treatment success" at the end of treatment course. In Table 2, it showed that patients with higher education level were more likely to achieve favourable outcomes (adjusted odds ratio [aOR]: 3.60, 95\% confidence interval $[C I]: 1.04-12.5)$. Such association was also observed in non-MDR-TB treatment group (aOR: 9.78, 95\% CI: 1.77-54.0) (Table 3).

\section{Discussion}

The MDR-TB epidemic remains a global public health problem, compromising the achievements in TB control in the past three decades. Over a two-year enrolment by performing DST to all culture-positive TB cases in four geographically varied regions of China, covering a population of 12 million, 58 (5.0\%) MDR-TB patients were detected from 1168 bacteriologically confirmed TB patients, which was far behind the estimation by the WHO. The results indicated that even with such a small amount of detected MDR-TB patients, most of them experienced major problems in obtaining timely MDR-TB diagnosis and appropriate treatment. More than half of patients had a delay longer than 90.0 days for receiving a DST report of MDR-TB, while only $32.8 \%$ were transferred to the up-level designated MDR-TB hospitals for MDR-TB treatment. Unfavourable outcomes occurred in $64.3 \%$ of diagnosed patients, resulting in a high risk of disease transmission in population as well as an increased risk of mortality.

The priorities of global MDR-TB control action ask for high-quality drug-susceptible TB treatment, rapid testing and detection of MDR/RR-TB, prompt access to 
Table 3 Risk factors for unfavourable treatment outcomes among the MDR-TB patients received non-MDR-TB treatment

\begin{tabular}{|c|c|c|c|c|}
\hline \multirow[t]{2}{*}{ Factors } & \multicolumn{2}{|c|}{ Patients with non-MDR-TB treatment } & \multirow{2}{*}{$\begin{array}{l}\text { Odds ratio } \\
\text { (95\% Cl) }\end{array}$} & \multirow{2}{*}{$\begin{array}{l}\text { Adjusted odds } \\
\text { ratio }(95 \% \text { Cl) }\end{array}$} \\
\hline & Favourable outcomes $(n=13)$ & Unfavourable outcomes $(n=24)$ & & \\
\hline Age (mean \pm SD) & $43.2 \pm 19.8$ & $55.1 \pm 14.1$ & $0.96(0.91-1.00)$ & \\
\hline Male & $12(92.3)$ & $22(91.7)$ & $1.09(0.09-13.3)$ & \\
\hline \multicolumn{5}{|l|}{ Study sites in } \\
\hline Western & $10(76.9)$ & $20(83.3)$ & 1 & \\
\hline Eastern & $3(23.1)$ & $4(16.7)$ & $1.50(0.28-8.04)$ & \\
\hline Body mass index (mean $\pm S D$ ) & $21.4 \pm 2.2$ & $20.1 \pm 2.2$ & $1.33(0.95-1.86)$ & $1.41(0.97-2.06)$ \\
\hline \multicolumn{5}{|l|}{ Education } \\
\hline Primary school or below & $4(30.8)$ & $20(83.3)$ & 1 & 1 \\
\hline Junior high school or above & $9(69.2)$ & $4(16.7)$ & $11.3(2.29-55.4)$ & $9.78(1.77-54.0)$ \\
\hline Married & $8(61.5)$ & $11(45.8)$ & $1.89(0.48-7.49)$ & $1.69(0.32-8.93)$ \\
\hline Health insurance & $13(100.0)$ & $23(95.8)$ & & \\
\hline Haemoptysis & $3(23.1)$ & $8(33.3)$ & $0.60(0.13-2.81)$ & $0.53(0.10-2.84)$ \\
\hline Tuberculosis treatment history & $4(30.8)$ & $14(58.3)$ & $0.32(0.08-1.33)$ & $0.15(0.02-1.05)$ \\
\hline Pulmonary cavities & $7(53.8)$ & $9(37.5)$ & $1.94(0.49-7.64)$ & $1.86(0.37-9.19)$ \\
\hline \multicolumn{5}{|l|}{ DST pattern } \\
\hline MDR & $7(53.8)$ & $9(37.5)$ & 1 & 1 \\
\hline Pre-XDR and XDR & $6(46.2)$ & $15(62.5)$ & $0.51(0.13-2.02)$ & $0.64(0.14-2.84)$ \\
\hline \multicolumn{5}{|l|}{ Strain family $(n=33)$} \\
\hline Non-Beijing family & $2(16.7)$ & $2(9.5)$ & 1 & 1 \\
\hline Beijing family & $10(83.3)$ & $19(90.5)$ & $0.53(0.06-4.32)$ & $0.90(0.08-10.2)$ \\
\hline \multicolumn{5}{|l|}{ Total delay (days) } \\
\hline$\leq 90.0$ & $9(69.2)$ & $12(50.0)$ & 1 & 1 \\
\hline$>90.0$ & $4(30.8)$ & $12(50.0)$ & $0.44(0.11-1.85)$ & $0.36(0.05-2.39)$ \\
\hline
\end{tabular}

DNA was failure to extract from six Mycobacterium tuberculosis isolates. Two patients were once reported as treatment success but were lost at the last yearly follow-up, thus excluded from the analysis. MDR Multidrug-resistant, SD Standard deviation, DST Drug susceptibility testing, XDR Extensively drug-resistant

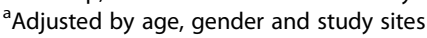

effective treatment and proper care, minimized risk of disease transmission and enhanced financing. The proportion of participants with MDR-TB diagnosis delay above 90.0 days was $51.7 \%$ in this study, lower than the 81.0\% reported in another Chinese study [8]. The difference might be explained by the delay caused by sending a positive mycobacterial culture to the provincial TB laboratory for DST in that study. Notably, $13.8 \%$ of the participants had a diagnosis delay over 180.0 days, almost the full duration of a drug-susceptible TB treatment course. To shorten the waiting time for MDR-TB diagnosis, rapid molecular diagnostics have been strongly recommended internationally [2]. As the country with the heaviest MDR-TB burden, India has made great efforts on the scale up of rapid molecular testing [18]. However, rapid molecular diagnostics weren't frequently used in China, with an approximate proportion of $15 \%$ in 2018 [2], because of their high cost and outof-pocket expenditures [19].
The poor accessibility of MDR-TB treatment even in detected MDR-TB patients also calls for immediate action. In South Africa, the treatment coverage for MDRTB patients reached around 70\% in 2018 [2], far above the accessibility shown in our study. The reason might be the high proportion of MDR-TB patients from our western study sites where accessibility to MDR-TB treatment was poorer than eastern regions [5]. Apart from geographic variations, the long waiting time for DST results is a probable cause leading to low coverage of MDR-TB treatment. In the non-MDR-TB treatment group, $15.4 \%$ of patients got their DST results after the completion of drug-susceptible TB treatment, posing a psychological barrier to motivate these patients to start a treatment course for MDR-TB. In addition, effective therapeutic effects of first-line drug regimen judged by clinicians or refusal of transferring to MDR-TB treatment made by patients were also the reasons causing low transfer rate to MDR-TB treatment. It indicated that health education of MDR-TB care should be enhanced 
for both clinicians and patients to improve their awareness.

A large proportion of patients ended up their treatment with loss to follow-up, treatment failure and incomplete treatment, thus amplifying the risk of MDR$\mathrm{TB}$ transmission. It may help to explain the findings in previous studies that over $70 \%$ of detected MDR-TB patients in China were caused by primary transmission [20, 21]. Similar results were also observed in our study that $56.9 \%(33 / 58)$ of MDR-TB patients did not have previous TB treatment history. After being transferred to MDR-TB treatment, $57.9 \%$ of patients were lost to follow-up or did not adhere to MDR-TB regimen. Previous studies indicated that reasons for loss during referral were inconvenient transportation, poor adherence of patients or foreseeable heavy economic burden [10, 22]. Furthermore, poor coordination between different sections under China's MDR-TB control program could be another reason, because the loss to follow-up occurred at the time of changing to outpatient treatment. To minimize the loss to follow-up and untreated cases, political commitment with financing should be prioritized and enhanced. The high cost for MDR-TB treatment $[23,24]$ made it both unaffordable and catastrophic for patients [10].

As expected, patients with MDR-TB but not receiving MDR-TB treatment regimen had a high proportion of unfavourable outcomes. Until the end of $2019,27.6 \%$ of them were confirmed with TB relapse while $13.8 \%$ had died within two years after treatment completion. It's an appalling but reasonable outcome due to the loss of the two most effective drugs [25], i.e. isoniazid and rifampicin, leaving only pyrazinamide and ethambutol as possible effective agents. Similar results were reported by other studies where MDR-TB patients with first-line TB treatment had high probability of TB relapse and death after the treatment completion [26, 27]. The possible explanation for the "treatment success" at the end of TB treatment might be the effect of pyrazinamide, an agent with strong sterilizing effect in the combination therapy $[25,28]$, and ethambutol. Another possible explanation is the lower proportion of mutations in katG/inhA loci in non-MDR-TB treatment group (62.9\%). The inconsistent between phenotypic and genotypic drug resistance indicated isoniazid might be an effective drug for some patients, highlighting the necessity of repeating DST and molecular diagnostics. However, ineffective treatment would increase the risk of developing additional drug resistance [29]. Providing first-line TB treatment for MDR-TB patients will most certainly promote further resistance to pyrazinamide and ethambutol.

A strength of this study is featured by the up to 6-year follow-up for the cohort, which could add valuable information on the experiences of MDR-TB patients on diagnosis and treatment, especially those ended up with treatment failure, incomplete treatment and loss to follow-up. Our study also has some limitations. Firstly, though mycobacterial culture and DST were given to both smear-positive patients and smear-negative patients with typical pulmonary lesions on chest X-ray, the rest smear-negative TB patients without culture confirmation could lead to underestimation of total number of MDRTB patients, even if it might be a very small amount. The reasons for not providing mycobacteria culture to all the smear-negative TB patients were the huge workloads and capacity constrains in designated county or district TB clinics, and the high uncertainty in diagnosing smear-negative TB patients. In this study, we only included clinically highly suspected smear-negative TB patients with typical pulmonary lesions on chest X-ray for sputum culture. Secondly, the small sample size limited the power of association analysis on unfavourable outcomes, though a two-year enrolment was given in four Chinese cities. Thirdly, treatment success in nonMDR-TB treatment group was still not well clarified. However, we believe that priority should be made for the provision of high-quality MDR-TB care for detected MDR-TB patients. Finally, patients remained in the treatment success at the last follow-up and those lost to follow-up were not verified by the bacteriological confirmation, which might lead to the underestimation of TB relapse. The high incidence of TB relapse in these patients should be enough to prove the ineffectiveness of treating MDR-TB patients with first-line TB drugs.

\section{Conclusions}

It is crucial for China's MDR-TB control program to establish high quality of care to all detected MDR-TB patients, especially in less developed regions. Our study showed that delayed MDR-TB diagnosis resulted in poor quality of MDR-TB care. Meanwhile, treating MDR-TB patients with a first-line drug regimen was found to be ineffective and led to a high incidence of unfavourable outcomes. It also constituted a risk for acquisition of additional drug resistance, especially in cases where a FQ was added to a failing regimen. The large proportion of loss to follow-up and untreated cases was a risk factor for increased disease transmission in population, as well as increased mortality among patients. Our findings highlight the urgency of strengthening patient management and financial support.

\section{Abbreviations}

MDR-TB: Multidrug-resistant tuberculosis; WHO: World Health Organization; RR: Rifampicin-resistant; DOTS: Directly observed treatment, short course strategy; DST: Drug susceptibility testing; M.tb: Mycobacteria tuberculosis; CDC: Center for Disease Control and Prevention; LJ: Loewenstein-Jenson; CTAB: Cetyl Trimethyl Ammonium Bromide; FQ: Fluoroquinolone;

SLID: Second-line injectable drugs; pre-XDR: Pre-extensively drug-resistant 


\section{Acknowledgements}

Not applicable.

\section{Authors' contributions}

$\mathrm{QZ}, \mathrm{YH}, \mathrm{VD}, \mathrm{SH}$, and BX designed the study. XZ, QZ, YH, WJ, and BX did the epidemiological investigations. XZ, YH, and WJ did the laboratory work and generated the laboratory data. XZ, and BX did the data analyses and drafted the manuscript. QZ, YH, VD, JB, and SH revised the manuscript. All authors read and approved the final manuscript.

\section{Funding}

This study was supported by the Swedish Research Council (No. 540-20138797 to Sven Hoffner) and the National Natural Science Foundation of China (No. 81361138019 to Biao Xu) joint project (VR-NSFC). The funders of the study had no role in study design, data collection, data analysis, data interpretation, or writing of the manuscript.

\section{Availability of data and materials}

The datasets used and analysed during the current study are available from the corresponding author on reasonable request.

\section{Ethics approval and consent to participate}

The study was approved by the Medical Institutional Review Board of the School of Public Health of Fudan University (approval No. IRB 2015-09-0565) and written informed consent was received from each participant.

\section{Consent for publication}

Not applicable.

\section{Competing interests}

The authors declare that they have no competing interests.

\section{Author details}

'Department of Epidemiology, School of Public Health and Key Laboratory of Public Health Safety (Ministry of Education), Fudan University, 130 Dong An Road, Shanghai 200032, China. ${ }^{2}$ Department of Global Public Health, Karolinska Institutet, Stockholm, Sweden. ${ }^{3}$ Department of Infectious Diseases, Karolinska University Hospital, Stockholm, Sweden. ${ }^{4}$ Division of Infectious Diseases, Department of Medicine Solna, Karolinska Institutet, Stockholm, Sweden.

Received: 6 May 2020 Accepted: 9 July 2020

Published online: 18 July 2020

\section{References}

1. Lange C, Chesov D, Heyckendorf J, Leung CC, Udwadia Z, Dheda K. Drugresistant tuberculosis: an update on disease burden, diagnosis and treatment. Respirology. 2018:23(7):656-73.

2. WHO. Global Tuberculosis Report 2019 Geneva: world health organization; 2019 [updated 17 October 2019. Available from: https://www.who.int/tb/ publications/global_report/en/.

3. WHO. MDR-TB factsheet 2018 Geneva 2018 [updated 2018. Available from: https://www.who.int/tb/areas-of-work/drug-resistant-tb/MDR-RR_TB_ factsheet_2018_Apr2019.pdf?ua=1.

4. Murray CJ, Ortblad KF, Guinovart C, Lim SS, Wolock TM, Roberts DA, et al. Global, regional, and national incidence and mortality for HIV, tuberculosis, and malaria during 1990-2013: a systematic analysis for the global burden of disease study 2013. Lancet. 2014;384(9947):1005-70.

5. Long O Qu Y, Lucas H. Drug-resistant tuberculosis control in China: progress and challenges. Infect Dis Poverty. 2016;5:9.

6. Wang L, Liu J, Chin DP. Progress in tuberculosis control and the evolving public-health system in China. Lancet. 2007;369(9562):691-6.

7. Zhou C, Jiang W, Yuan L, Lu W, He J, Zhao Q, et al. Access to bacteriologicbased diagnosis in smear positive retreatment tuberculosis patients in rural China: a cross-sectional study in three geographic varied provinces. PLoS One. 2016;11(1):e0146340.

8. Zhang XL, Yin J, Li HT, Li SG, Walley J, Zou GY, et al. Diagnostic and treatment delays of multidrug-resistant tuberculosis before initiating treatment: a crosssectional study. Tropical Med Int Health. 2015;20(11):1431-7.

9. Yang Z, Zhou C, Ning Z, Lu W, Zhao Q, Hu Y, et al. High-risk screening and detection of multidrug-resistant tuberculosis in two prefectures of China: a drug susceptibility surveillance-based secondary data analysis. Glob Health Action. 2018:11(1):1500763.

10. Chen Y, Zhao Y. Multidrug-resistant tuberculosis in rural China: lack of public awareness, unaffordable costs and poor clinical management. Case Reports. 2018;2018:bcr-2018-225794

11. Zhang Y, Wu S, Xia Y, Wang N, Zhou L, Wang J, et al. Adverse events associated with treatment of multidrug-resistant tuberculosis in China: an ambispective cohort study. Med Sci Monit. 2017;23:2348-56.

12. Liu Z, Pan A, Wu B, Zhou L, He H, Meng Q, et al. Feasibility of a new model for early detection of patients with multidrug-resistant tuberculosis in a developed setting of eastern China. Tropical Med Int Health. 2017;22(10): 1328-33.

13. Wang Y. Guidelines for the prevention and control of multidrug-resistant tuberculosis. Beijing: Military Science Publishing House; 2012.

14. WHO. WHO consolidated guidelines on drug-resistant tuberculosis treatment Geneva: World Health Organization; 2019 [Available from: https:/www.who.int/ tb/publications/2019/consolidated-guidelines-drug-resistant-TB-treatment/en/.

15. WHO. Policy guidance on drug-susceptibility testing (DST) of second-line antituberculosis drugs Geneva: World Health Organization; 2008 [updated 2008. Available from: https://www.who.int/tb/publications/2008/ whohtmtb_2008_392/en/.

16. WHO. Definitions and reporting framework for tuberculosis Geneva 2013 [updated December 2014. Available from: https://www.who.int/tb/ publications/definitions/en/.

17. Zignol M, Cabibbe AM, Dean AS, Glaziou P, Alikhanova N, Ama C, et al. Genetic sequencing for surveillance of drug resistance in tuberculosis in highly endemic countries: a multi-country population-based surveillance study. Lancet Infect Dis. 2018;18(6):675-83.

18. TBC India. India TB report 2018 New Delhi: Central TB Division; 2018 [Available from: https://tbcindia.gov.in/showfile.php?lid=3314.

19. Tang S, Wang L, Wang H, Chin DP. Access to and affordability of healthcare for TB patients in China: issues and challenges. Infect Dis Poverty. 2016;5:10.

20. Yang C, Luo T, Shen X, Wu J, Gan M, Xu P, et al. Transmission of multidrugresistant Mycobacterium tuberculosis in Shanghai, China: a retrospective observational study using whole-genome sequencing and epidemiological investigation. Lancet Infect Dis. 2017;17(3):275-84.

21. Zhao YL, Xu SF, Wang LX, Chin DP, Wang SF, Jiang GL, et al. National survey of drug-resistant tuberculosis in China. N Engl J Med. 2012;366(23):2161-70.

22. Yin J, Wang $X$, Zhou $L$, Wei $X$. The relationship between social support, treatment interruption and treatment outcome in patients with multidrugresistant tuberculosis in China: a mixed-methods study. Tropical Med Int Health. 2018:23(6):668-77.

23. Lunte K, Cordier-Lassalle T, Keravec J. Reducing the price of treatment for multidrug-resistant tuberculosis through the global drug facility. Bull World Health Organ. 2015;93(4):279-82.

24. Floyd K, Hutubessy R, Kliiman K, Centis R, Khurieva N, Jakobowiak W, et al. Cost and cost-effectiveness of multidrug-resistant tuberculosis treatment in Estonia and Russia. Eur Respir J. 2012;40(1):133-42.

25. Svensson EM, Svensson RJ, Te Brake LHM, Boeree MJ, Heinrich N, Konsten S, et al. The potential for treatment shortening with higher rifampicin doses: relating drug exposure to treatment response in patients with pulmonary tuberculosis. Clin Infect Dis. 2018;67(1):34-41.

26. He GX, Xie YG, Wang LX, Borgdorff MW, van der Werf MJ, Fan JH, et al. Follow-up of patients with multidrug resistant tuberculosis four years after standardized first-line drug treatment. PLoS One. 2010:5(5):e10799.

27. Chiang CY, Enarson DA, Yu MC, Bai KJ, Huang RM, Hsu CJ, et al. Outcome of pulmonary multidrug-resistant tuberculosis: a 6-yr follow-up study. Eur Respir J. 2006;28(5):980-5.

28. Pasipanodya JG, Mcllleron H, Burger A, Wash PA, Smith P, Gumbo T. Serum drug concentrations predictive of pulmonary tuberculosis outcomes. $J$ Infect Dis. 2013;208(9):1464-73.

29. Cegielski JP, Kurbatova E, van der Walt M, Brand J, Ershova J, Tupasi T, et al. Multidrug-resistant tuberculosis treatment outcomes in relation to treatment and initial versus acquired second-line drug resistance. Clin Infect Dis. 2016;62(4):418-30 